\title{
Efficacy and safety of sphenopalatine artery (SPA) ligation and SPA embolisation: a 9-year review of management of epistaxis in a district general teaching hospital
}

Miss Kimberley Lau, Mr Mahmoud Daoud, Mr Ullas Raghavan, Mr Aftab Ahmed, Mr Sidhartha Nagala Doncaster Royal Infirmary, Doncaster and Bassetlaw Teaching Hospitals NHS Foundation Trust, UK

\section{BACO International 2018, Manchester UK}

\section{Background}

The incidence of epistaxis is about $\mathrm{I} / \mathrm{I} 000$ per year (I). The management of posterior epistaxis remains a challenging problem for the ENT surgeon. The re-bleed rate for SPA ligation and embolization has been quoted in literature to be $4-33 \%$ and $13-29 \%(2,3)$ and both procedures are accompanied with their own risks.

\section{Objectives}

This retrospective study aimed to identify all epistaxis admissions in 9 years and to determine the efficacy and complication rates of SPA intervention.

\section{Methods}

Inpatient admission data (Jan 2008-Mar 2015) for epistaxis was obtained from the hospital audit department. All SPA intervention procedures were identified from electronic theatre records and radiology database. Case notes were reviewed.

\section{Results}

There were 1859 epistaxis inpatient admissions (I I I were readmission episodes). 65 patients required SPA intervention. $45 \%$ of epistaxis readmissions required SPA intervention.

\section{Conclusions}

It is important to consider re-bleed and complication rates when gaining informed consent from patients for any SPA interventions.

Table 1. SPA Ligation results
\begin{tabular}{|c|c|}
\hline $\begin{array}{c}\text { Total SPA ligation procedures }=\mathbf{5 4} \\
\text { Mean Age }=58.4 \quad \text { Male: Female }=1.16: 1\end{array}$ \\
\hline Bilateral SPA ligation & $16 / 54(30 \%)$ \\
\hline Right SPA ligation & $22 / 54(40 \%)$ \\
\hline Left SPA ligation & $16 / 54(30 \%)$ \\
\hline
\end{tabular}

Table 2. Post-SPA ligation re-bleed rates
\begin{tabular}{|c|c|}
\hline $\begin{array}{c}\text { Major epistaxis requiring revision procedures } \\
\text { (futher ligation or embolisation) }\end{array}$ & $11 / 54(20 \%)$ \\
\hline $\begin{array}{c}\text { Major epistaxis unresolved } \\
\text { (requiring referral to tertiary centre) }\end{array}$ & $1 / 54(1.9 \%)$ \\
\hline $\begin{array}{c}\text { Minor epistaxis resolved } \\
\text { (no further intervention required) }\end{array}$ & $8 / 54(14.8 \%)$ \\
\hline Total re-bleed rate & $\mathbf{2 0 / 5 4 ( 3 7 \% )}$ \\
\hline
\end{tabular}

Table 3. Post-SPA ligation complications
\begin{tabular}{|l|ll|}
\hline Major & HDU admission for chest pain - 1/54 (1.9\%) \\
\hline Minor & - & Local Infection: $2 / 54(3.7 \%)$ \\
& - & Pneumonia: $1 / 54(1.9 \%)$ \\
& & Alar necrosis: $1 / 54(1.9 \%)$ \\
\hline
\end{tabular}

\begin{tabular}{|c|c|}
\hline Total SPA ligation procedures $=11$ & \\
\hline Male: Female $=1.75: 1$ & \\
\hline Bilateral SPA embolisation & $4 / 11(36 \%)$ \\
\hline Right SPA embolisation & $3 / 11(27 \%)$ \\
\hline Unknown side embolization (not found in notes) & $1 / 11(9 \%)$ \\
\hline Failed/abandoned procedure & $\begin{array}{c}3 / 11 \text { (36\%) } \\
\text { (2 intra-op CVA, } 1 \\
\text { difficult anatomy) }\end{array}$ \\
\hline
\end{tabular}

Table 5. Post-SPA embolisation re-bleed rates
\begin{tabular}{|c|c|}
\hline $\begin{array}{c}\text { Major epistaxis requiring revision procedures } \\
\text { (futher ligation or embolisation) }\end{array}$ & $1 / 8(12.5 \%)$ \\
\hline $\begin{array}{c}\text { Minor epistaxis resolved } \\
\text { (no further intervention required) }\end{array}$ & $3 / 8(37.5 \%)$ \\
\hline Total re-bleed rate & $4 / 8(50 \%)$ \\
\hline
\end{tabular}

Table 6. Post-SPA embolisation complications
\begin{tabular}{|l|l|}
\hline Major & $\begin{array}{l}\text { Peri-procedure CVA }: 2 / 11(18 \%) \\
\text { ( } 1 \text { full recovery within } 24 \text { hours, } 195 \% \text { recovery })\end{array}$ \\
\hline Minor & $\bullet$ None \\
\hline
\end{tabular}

